\title{
A telemetric study of physiologic changes in mice with induced autoimmune encephalomyelitis
}

\author{
Abigail C. Buenafe, $P h D^{1}$, Heather Zwickey, $P h D^{2}$, Nicole Moes, $M S^{3}$, Barry Oken, $M D^{1}$ \& \\ Richard E. Jones, $P h D^{1,3}$
}

Dysfunction of the autonomic nervous system may be an important component of disease progression in multiple sclerosis (MS), a paralytic inflammatory autoimmune disease of the central nervous system. Using the experimental autoimmune encephalomyelitis mouse model of MS, the authors carried out a pilot study to investigate whether telemetric monitoring might be a feasible approach for detecting disturbances in the autonomic control of heart rate and blood pressure after disease induction. Telemetric monitoring devices that were implanted in mice provided useful information regarding the physiologic changes that accompanied disease induction and progression. Changes were observed in heart rate, blood pressure, heart rate variability and diurnal rhythm immediately before and after disease onset. The device implantation procedure did not seem to alter the course of disease. Further investigation may establish these methods as a system for studying the relationships between MS progression and autonomic regulation of physiological status.

The autonomic nervous system regulates internal homeostasis. This regulatory process is functionally mediated by the sympathetic and parasympathetic divisions of the nervous system, which generally work in opposition to each other. Sympathetic functions typically enable 'fight or flight' responses, whereas the parasympathetic division is more involved in 'rest and digest' functions of target organs. Fluctuations in heart rate (HR), for example, are influenced by both the sympathetic and parasympathetic pathways, and spectral analysis of heart rate variability (HRV) can distinguish components contributed by each pathway ${ }^{1,2}$. In humans, decreased HRV is currently used as a predictor of increased morbidity and mortality with various forms of cardiovascular ${ }^{3-5}$ and non-cardiovascular diseases ${ }^{6,7}$. Direct innervation of immune system components has been demonstrated for the sympathetic pathway but not for the parasympathetic pathway ${ }^{8}$; however, there is much evidence to suggest that the parasympathetic nervous system down-modulates the immune response $\mathrm{e}^{9,10}$.
It is not known exactly how the autonomic nervous system responds to and is affected by the initiation and progression of neuroimmunological disease. Multiple sclerosis (MS) is a neurodegenerative disease caused by immune effector cell insult on the myelin sheath of the central nervous system. MS can be associated with a monophasic, relapsing or chronic disease course. There have been numerous reports of autonomic and cardiovascular dysfunction associated with the disease $\mathrm{e}^{11-14}$. In some cases, autonomic dysfunction has been correlated with disease status and fatigue in people with $\mathrm{MS}^{15-17}$. Other studies have noted a correlation in MS patients between cardiovascular autonomic dysfunction and midbrain or brainstem lesions observed by magnetic resonance imaging ${ }^{18-20}$. Spectral analyses of HRV in individuals with MS have indicated disturbances in both parasympathetic and sympathetic pathways ${ }^{21-23}$. Researchers attempting to study the involvement of autonomic disturbance in MS

${ }^{1}$ Department of Neurology, Oregon Health and Science University, Portland, OR 97239. ${ }^{2}$ Helfgott Research Institute, National College of Natural Medicine, Portland, OR 97201. ${ }^{3}$ Neuroimmunology Research, Veterans Affairs Medical Center, Portland, OR 97239. Correspondence should be addressed to A.C.B. (buenafea@ohsu.edu) or R.E.J. (jonesric@ohsu.edu). 
have encountered various difficulties related to the unpredictability and extended length of the disease course, as well as problems associated with continuous monitoring.

Experimental autoimmune encephalomyelitis (EAE) serves as a mouse model of MS. In SJL mice, EAE is induced by immunization with a peptide sequence derived from region 139-151 of proteolipid protein (PLP139-151), a component of the myelin sheath $^{24}$. Alternatively, EAE may also be induced in the SJL mouse strain by the passive transfer of PLP139-151specific $\mathrm{T}$ cells ${ }^{24,25}$. Implantable telemetric devices that record physiological data such as $\mathrm{HR}$, blood pressure (BP), activity and body temperature in freely moving rodents ${ }^{26,27}$ can be used to monitor physiological changes during the EAE disease course.

The murine HR can range from 400 to 800 beats per min, approximately 10 times faster than the human HR. Quantitative analysis of the beat-to-beat fluctuations in the murine HR is a valid means of measuring cardiac autonomic regulation ${ }^{28,29}$. The murine respiratory rate, at 250-350 breaths per min, is also 10 times greater than the human respiratory rate at rest and affects sinus arrhythmia, which in turn influences HRV. HR and HRV are dependent on activity level and circadian rhythm; in addition, HRV is influenced by characteristics such as age, gender and mouse strain ${ }^{30,31}$.

Our goal in this pilot study was to establish the feasibility of using telemetric monitoring to collect continuous physiological data in EAE mice. We further wished to investigate the potential of using HR, BP and HRV in EAE as a means to better understand the status of the autonomic nervous system during the development and progression of autoimmune disease in the central nervous system. To this end, we carried out telemetric monitoring in SJL mice that were passively induced to develop a relapsing-remitting EAE disease course.

\section{METHODS}

Mice

We obtained female SJL mice from the Jackson Laboratory (Bar Harbor, ME). Mice were 18 weeks old at the time of device implantation or sham surgery. Mice were housed in sterilized cages and bedding under specific pathogen-free conditions at the Veterinary Medical Unit of the Portland Veterans Affairs Medical Center. Conditions were monitored using sentinel mice that were exposed to pooled dirty bedding for $>28 \mathrm{~d}$ and then tested serologically for multiple pathogens and parasites. At the time of this investigation, sentinel mice were determined to be free of Sendai virus, mouse parvovirus, minute virus of mice, reovirus type 3 , pneumonia virus of mice, mouse coronavirus, Mycoplasma pulmonis, mouse rotavirus, mouse encephalomyelitis virus and rodent pinworms and mites. All procedures were approved by our IACUC and carried out in accordance with institutional guidelines.

\section{Telemetric monitoring}

Telemetry transmitter devices (model PA-C10, Data Sciences International, St. Paul, MN) were implanted into mice that were anesthetized with isoflurane. Per supplier recommendations, we inserted the tip of the device's catheter into the left carotid artery of the mouse. We then placed the transmitter body subcutaneously (s.c.) in an abdominal pocket, sutured incisions and administered buprenorphine to the mouse as an analgesic.

We carried out a preliminary experiment in healthy mice to evaluate the implantation and monitoring procedures. Device-implanted mice $(n=5)$ recovered from surgery in single housing for 1 week under a standard light:dark cycle of $12 \mathrm{~h}: 12 \mathrm{~h}$. Mice were then transferred to paired housing or remained in single housing for monitoring of HR, BP and activity.

In a second experiment, we implanted telemetry transmitter devices into another group of mice $(n=5)$ in which EAE would later be induced. For this experiment, additional mice $(n=5)$ underwent a sham procedure in which the carotid artery was exposed but no device was implanted. One person carried out all implantation and sham surgeries over 2 consecutive d. Mice recovered from surgery for 1 week as described above. After recovery, we transferred each device-implanted mouse to paired housing with a sham-treated mouse and monitored $\mathrm{HR}, \mathrm{BP}$ and activity of implanted mice for 1 week. EAE was later induced only in mice with stable BP.

Transmitters were turned on magnetically during recovery. Receiver platforms beneath mouse cages transmitted the radiofrequency data at $500 \mathrm{~Hz}$ to a data-exchange matrix for conversion and storage on the hard drive of a personal computer. Three days before EAE induction, we programmed the data collection system (Dataquest A.R.T. software, version 4.0, Data Sciences International, St. Paul, MN) to record data once per min for $10 \mathrm{~s}$.

Fluctuations in HR are influenced by both sympathetic and parasympathetic pathways of the autonomic nervous system. To maximize detection of the parasympathetic contribution to HRV, we collected continuous waveform data for the analysis of HRV during rest. We suspended scheduled intermittent data collection each day towards the end of the low activity (rest) cycle, between 12:00 PM and 2:00 PM (zeitgeber time (ZT) 6 and ZT8, where ZT0 is the start of the light cycle at 6:00 AM). At these times, we collected continuous waveform data for 7-10 min.

\section{HRV analysis}

$\mathrm{BP}$ waveform data were processed in segments of $60 \mathrm{~s}$ to allow analysis of at least 500 consecutive beats per segment. Using the system's software, we extracted inter-beat interval data from the waveform data for the time domain analysis of HRV. For frequency domain analysis, inter-beat 

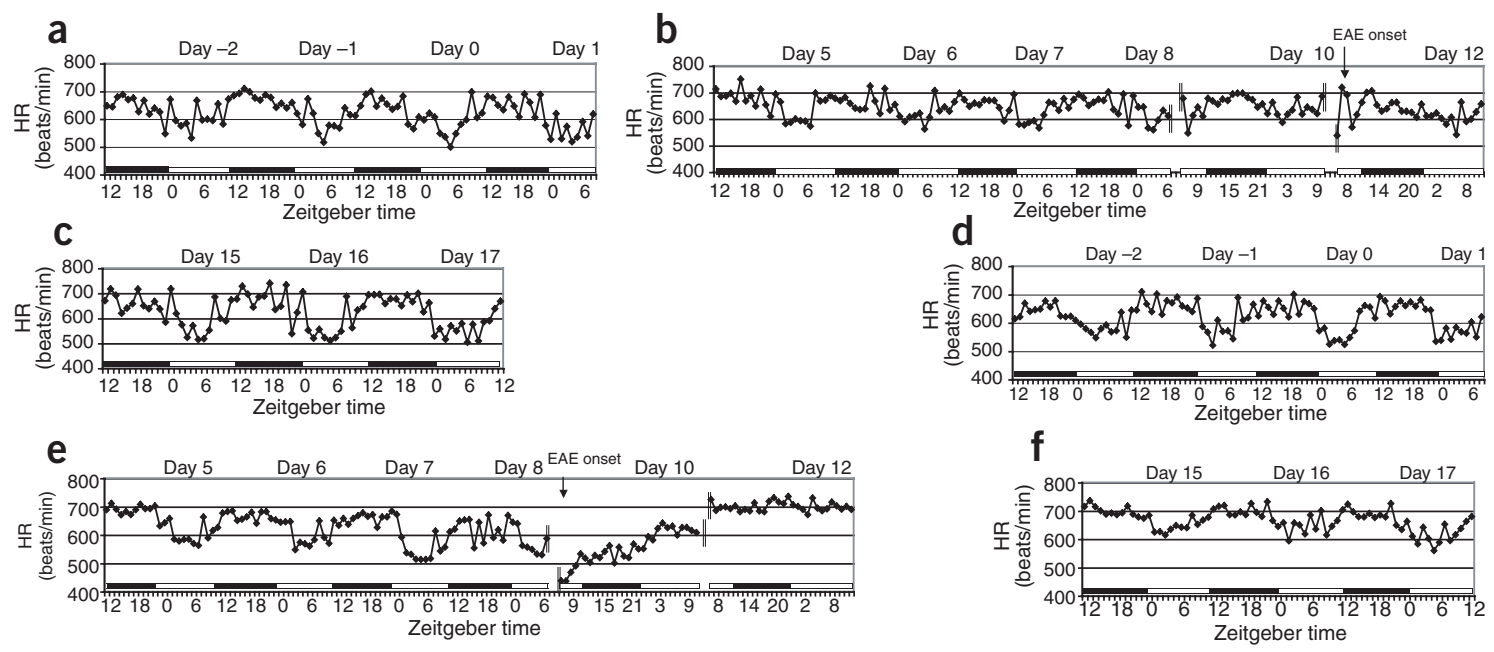

FIGURE 1 | Continuous trace of HR during the pre-disease, disease onset and recovery periods of EAE in mice implanted with telemetry monitoring devices. Black and white bars indicate the dark and light phases, respectively. Day 0 indicates day of T-cell transfer for EAE induction. (a-c) Mouse 2. (d-f) Mouse 4.

interval data were interpolated at $50 \mathrm{~Hz}$, detrended and mean-suppressed. We then applied the periodogram function for the estimation of power spectral density. We set the low frequency range at $0.4-1.5 \mathrm{~Hz}$ and the high frequency range at $1.5-4.0 \mathrm{~Hz}$. We determined normalized frequencies by dividing low frequency or high frequency by the total power (0.015-4.0 Hz; refs. 29,32).

\section{Induction and scoring of EAE}

We induced EAE in device-implanted $(n=2)$ and sham-treated $(n=5)$ SJL mice by intraperitoneal (i.p.) injection with $7.5 \times 10^{6}$ PLP139-151-specific $\mathrm{T}$ cells. Mice began to show signs of paralysis 9-12 d after transfer, beginning with a limp tail and then progressing with ascending paralysis of the hind limbs and then forelimbs. The condition of each mouse was scored daily on a scale of 1-6 as follows: 0 for normal condition; 1 for limp tail or mild hind limb weakness; 2 for limp tail and moderate hind limb weakness or mild ataxia; 3 for limp tail and moderately severe hind limb weakness; 4 for limp tail and severe hind limb weakness, mild forelimb weakness or moderate ataxia; 5 for limp tail and paraplegia with no more than moderate forelimb weakness; and 6 for limp tail and paraplegia with severe forelimb weakness or severe ataxia or moribund condition. At the first indication of disease, we provided softened food in a small dish on the cage bedding material. Mice with a score of 4 or higher received fluids i.p. (sterile $5 \%$ dextrose in saline, $1 \mathrm{ml}$ per d). During daily evaluation, we also weighed each mouse to ensure that its weight had not decreased by more than 20\% from the baseline weight recorded before disease induction. Although such a situation did not arise in this study, weight loss of $20 \%$ or more within 3 consecutive d would indicate that a humane endpoint (euthanasia) had been reached for that mouse.

\section{Generation of PLP139-151-specific T cell lines}

We actively immunized female SJL mice s.c. at four sites distributed across the lower back and flanks with $150 \mu \mathrm{g}$ PLP139-151 peptide (HCLGKWLGHPDKF) in $0.2 \mathrm{ml}$ complete Freund's adjuvant containing Mycobacterium tuberculosis ( $50 \mu \mathrm{l}$ per injection). We harvested spleens and lymph node cells $10 \mathrm{~d}$ after immunization and processed them to a single-cell suspension. We lysed red blood cells with RBC Lysis buffer (Sigma, St. Louis, $\mathrm{MO})$. Cells were stimulated at $4 \times 10^{6}$ cells per $\mathrm{ml}$ in stimulation medium (Roswell Park Memorial Institute (RPMI) 1640 containing $1 \mathrm{mM}$ sodium pyruvate, $2 \mathrm{mM}$ L-glutamine, 57.2 $\mu \mathrm{M} 2$-mercaptoethanol and 10\% fetal bovine serum) containing $10 \mu \mathrm{g}$ PLP139-151 peptide for $72 \mathrm{~h}$. We then expanded T cells in growth medium (RPMI 1640 containing $1 \mathrm{mM}$ sodium pyruvate, $2 \mathrm{mM}$ L-glutamine, 57.2 $\mu \mathrm{M}$ 2-mercaptoethanol, 10\% fetal bovine serum and $20 \mathrm{U} / \mathrm{ml}$ recombinant interleukin-2) for $5 \mathrm{~d}$ before freezing and storing them in liquid nitrogen. Before passive transfer, we thawed and stimulated cells for $72 \mathrm{~h}$ with PLP139-151 peptide in the presence of irradiated thymocytes and splenocytes as antigen-presenting cells.

\section{RESULTS}

\section{Telemetric monitoring in healthy mice}

Before beginning telemetric monitoring in EAE mice, we evaluated the implantation and monitoring procedures in healthy mice $(n=5)$. One mouse died of unknown causes 4 weeks after surgery, and in another mouse, signal transmission was erratic. We excluded data from these two mice from the experiment. After the three remaining mice recovered from surgery, we housed two device-implanted mice together. We collected telemetry data from only one of these mice, 
as data transmission can be captured from only one mouse at a time per cage. The third implanted mouse was housed singly for data collection. We observed a diurnal pattern of HR, BP and activity corresponding to the light:dark cycle in the two healthy mice that we monitored (data not shown).

\section{Telemetric monitoring in EAE mice}

In a second experiment, we telemetrically monitored mice in which EAE was later induced. The telemetry device was successfully implanted in three of the five mice in which implantation was attempted. Two of the five mice did not survive implantation owing to rupture of the carotid artery during surgery.

Two weeks after implantation surgery, we induced EAE in device-implanted mice $(n=2)$ and in sham-treated mice $(n=5)$. We induced EAE in only two of the three mice that had been successfully implanted with transmitter devices, because one mouse was euthanized $10 \mathrm{~d}$ after implantation because of self-inflicted wounding (gnawing at the transmitter battery site).

Both device-implanted mice and three of the five sham-treated mice developed EAE. The incidence rate of disease observed in this experiment $(71 \%)$ was within the range observed in previous experiments. Thus, it is not possible in the current study to draw conclusions regarding the influence of the surgical procedure or device implantation on disease incidence. Disease course and severity in sham-treated mice were qualitatively similar to those in the implanted mice, in that similar and variable disease patterns developed in both groups (data not shown).

\section{HR in EAE mice}

As noted above, before inducing EAE (day 0) we readily observed a diurnal rhythm in the HR of both device-implanted mice (hereafter referred to as mouse 2 and mouse 4; Fig. 1a,d). Consistent with a nocturnal activity pattern, peak levels in HR were associated with the dark phase, and trough levels were associated with the light phase.

In mouse 2, we observed a loss of amplitude in the diurnal HR pattern, equivalent to a rise in HR during rest (light period; Fig. 1b), as early as day 5 , though signs of motor dysfunction were not observed until day 11. In mouse 4 , we observed disruptions in the diurnal HR upon onset of motor dysfunction on day 9 (Fig. 1e). In this mouse, the diurnal HR pattern was completely disrupted and an initial severe drop in HR was followed by an elevation in HR that was sustained throughout the light:dark cycle. Mouse 2 developed moderate EAE, which reached a peak score of 3.0 on day 12 (Fig. 2a). Disease ameliorated after day 14 and dropped to a score of 1.0 by day 17 . The EAE disease course in mouse 4 (Fig. 2b) was more severe than that in mouse 2 (Fig. 2a), reaching a peak score of 5.0 on day 11 and
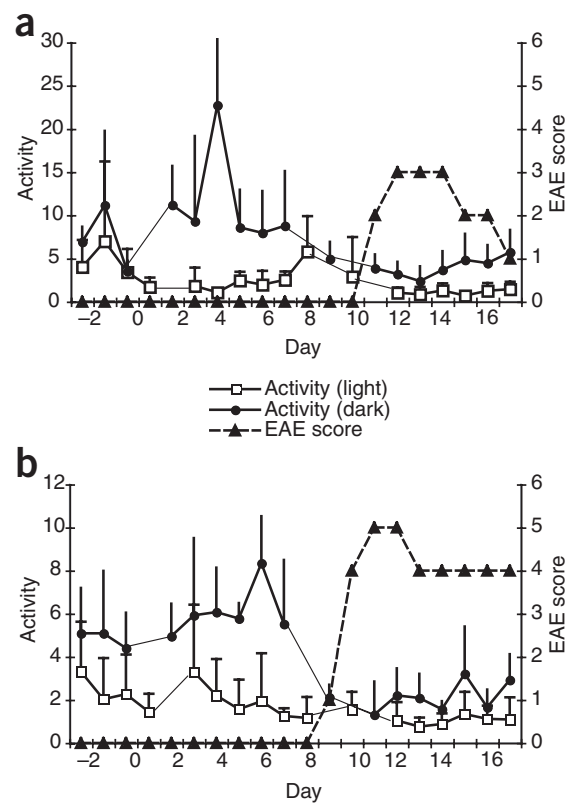

FIGURE 2 | Activity level and EAE disease score in deviceimplanted mice at trough levels during the light phase and at peak levels during the dark phase. Day 0 indicates day of T-cell transfer for EAE induction. Bars indicate s.d. (a) Mouse 2. (b) Mouse 4.

dropping to a score of 4.0 on day 13 . This score was retained until the last day of the experiment (day 17). The diurnal HR for both mice began to approach pre-disease amplitude after days 15-17 (Fig. 1c,f).

\section{$B P$ and motor activity in EAE mice}

We monitored BP continuously before and after disease induction in both device-implanted mice and observed patterns similar to those observed in the HR (Fig. 3). The diurnal pattern of BP in mouse 2 did not begin to approach pre-disease peak and trough levels until day 15 (Fig. 3c). In mouse 4, the diurnal pattern of BP seemed normal until disease onset (day 9), at which point BP became substantially elevated and remained high regardless of the phase in the light:dark cycle. BP levels began to decrease after day 15 but had not returned to the pre-disease pattern by day 17 (Fig.3d-f). Overall, the magnitude of change observed in $\mathrm{HR}$ and $\mathrm{BP}$ in association with disease onset, particularly during the light phase, as well as the magnitude of change in the diurnal pattern, seemed to correlate with the severity of disease. In mouse 2, which had relatively mild EAE (maximum score was 3.0), changes in HR and BP were less marked than in mouse 4, which had more severe EAE (maximum score was 5.0) and a longer duration of disease.

As expected, telemetric monitoring showed that after disease onset (motor dysfunction), activity decreased 

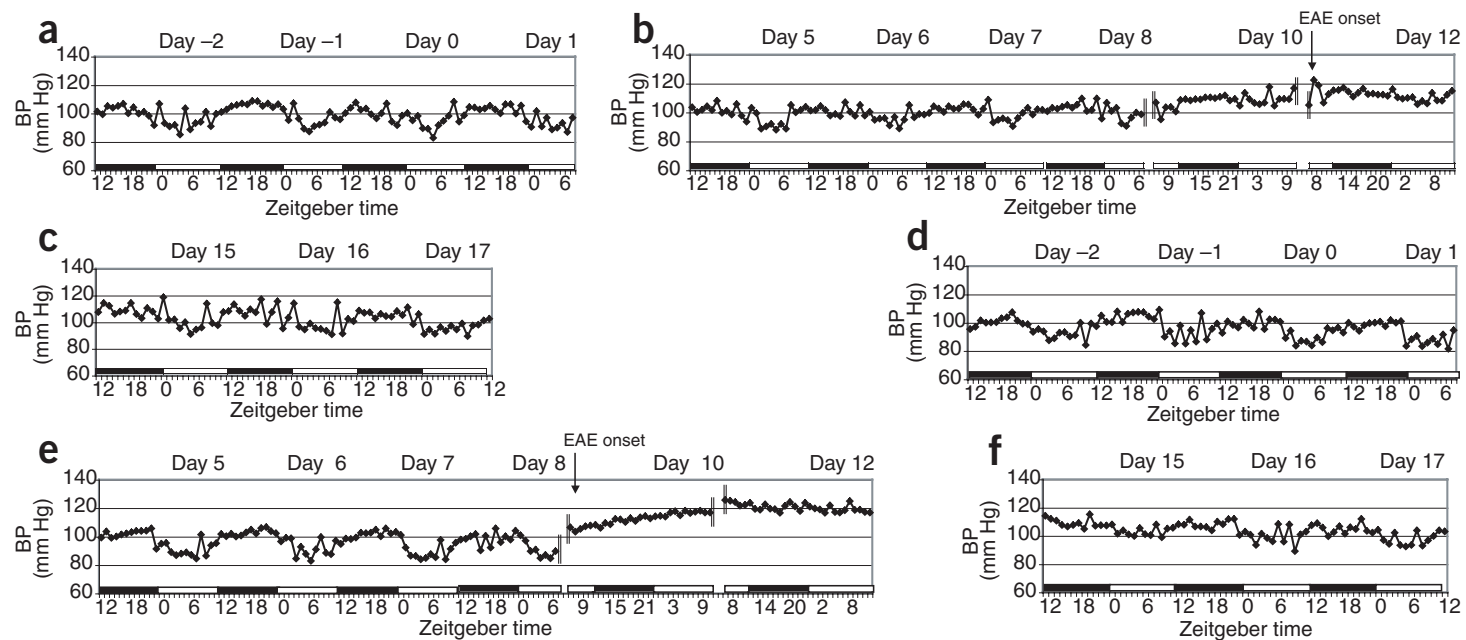

FIGURE 3 | Continuous trace of BP during the pre-disease, disease onset and recovery periods of EAE in mice implanted with telemetry monitoring devices. Black and white bars indicate the dark and light phases, respectively. Day 0 indicates day of T-cell transfer for EAE induction. (a-c) Mouse 2. (d-f) Mouse 4.

during the dark (active) period (Fig. 2). The relationship between motor activity and autonomic changes is not clear, but the decreased motor activity probably did not directly account for the observed increases in HR and BP.

\section{HRV in EAE mice}

We calculated changes in HRV before, during and after EAE onset in the frequency domain by power spectrum analysis of contributions of high frequency (influenced by parasympathetic inputs) and low

a

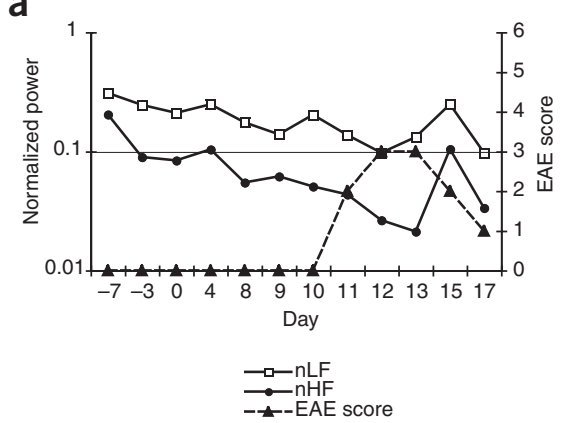

b

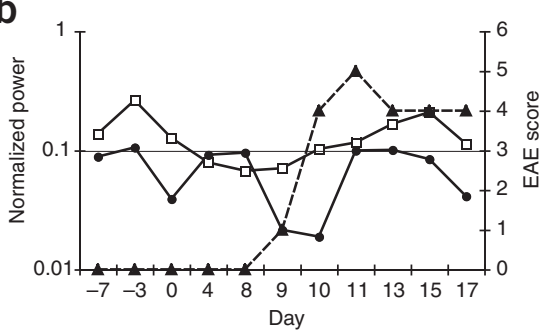

FIGURE 4 | Time domain analysis of HRV in device-implanted mice. We observed a loss of variability in the inter-beat interval immediately before or after EAE onset relative to predisease induction in the normalized low frequency (nLF) and normalized high frequency ( $\mathrm{nHF}$ ) ranges. Day 0 indicates day of T-cell transfer for EAE induction. (a) Mouse 2. (b) Mouse 4. frequency (influenced by both parasympathetic and sympathetic input; Fig. 4). Decreases in HRV occurred at both high and low frequency powers in both device-implanted mice, consistent with a loss of normal diurnal variation in HR, which is regulated by the autonomic nervous system. An observed decrease in the high or low frequency power spectrum indicates a change in autonomic function but does not determine whether sympathetic or parasympathetic activity is increased or decreased ${ }^{33}$.

Time domain analysis of the inter-beat intervals also showed a correlation between decreased HRV and EAE (Tables 1 and 2). In mouse 2, inter-beat intervals became shorter and HRV decreased on the day preceding disease onset (day 10). Mouse 4 showed an increase in inter-beat intervals with increased HRV on the day of onset (day 9), followed by low HRV for the remainder of the disease course. These changes are consistent with the respective HR patterns observed for each mouse during the disease course (Fig. 1) and support the hypothesis that changes in HRV may vary depending on the course and severity of disease.

\section{DISCUSSION}

The results of this pilot study demonstrate that mice implanted with a telemetry monitoring device can develop EAE, and that this monitoring method is feasible and useful in the EAE disease model. Device-implanted mice were able to recover from surgery, and the time course of autoimmunity induction in these mice was similar to that observed in sham-treated mice. We note that EAE mice did not seem to possess characteristics that would preclude data collection using the telemetric methods we have described.

HR and BP followed similar patterns, increasing during the dark cycle. Similarly, activity levels were 


\begin{tabular}{|c|c|c|c|c|c|c|}
\hline Day & EAE score & $\mathrm{RR}(\mathrm{ms}) \pm \mathrm{s} . \mathrm{d}$ & SDRR & $P_{\text {SDRR }}$ & CV $(\%) \pm$ s.d. & $P_{\mathrm{CV}}$ \\
\hline-3 & 0 & $99 \pm 7$ & $9 \pm 6$ & 0.12 & $9 \pm 6$ & 0.2 \\
\hline 0 & 0 & $103 \pm 12$ & $12 \pm 4$ & - & $12 \pm 4$ & - \\
\hline 4 & 0 & $93 \pm 3$ & $5 \pm 2$ & $<0.0001$ & $5 \pm 3$ & $<0.0001$ \\
\hline 8 & 0 & $104 \pm 3$ & $6 \pm 3$ & 0.0002 & $6 \pm 3$ & 0.0003 \\
\hline 9 & 0 & $114 \pm 4$ & $8 \pm 3$ & 0.01 & $7 \pm 3$ & 0.002 \\
\hline 10 & 0 & $82 \pm 1$ & $2 \pm 0.4$ & $<0.0001$ & $2 \pm 0.5$ & $<0.0001$ \\
\hline 11 & 2 & $114 \pm 4$ & $8 \pm 3$ & 0.007 & $7 \pm 3$ & 0.002 \\
\hline 12 & 3 & $105 \pm 9$ & $7 \pm 2$ & 0.0002 & $6 \pm 1$ & $<0.0001$ \\
\hline 13 & 3 & $120 \pm 3$ & $7 \pm 2$ & 0.0001 & $6 \pm 2$ & $<0.0001$ \\
\hline 15 & 2 & $99 \pm 13$ & $7 \pm 2$ & 0.0003 & $7 \pm 2$ & 0.0006 \\
\hline 17 & 1 & $120 \pm 7$ & $7 \pm 2$ & 0.0001 & $6 \pm 2$ & $<0.0001$ \\
\hline
\end{tabular}

highest during the dark phase and lowest during the light phase. These results indicate that our approach to physiological monitoring in mice can detect diurnal variations in $\mathrm{HR}, \mathrm{BP}$ and activity that are consistent with nocturnal behavioral patterns reported previously ${ }^{30}$.

We noted a disruption of normal HR and BP patterns in mice immediately before and after the onset of passively induced EAE. In mouse 4 , in which EAE was relatively severe, $\mathrm{HR}$ remained elevated for much longer after disease onset compared with the HR of mouse 2, which had moderate EAE. In mouse 2, HR peaked close to disease onset but then quickly returned to normal levels. This indicates that the pre-disease diurnal pattern can re-emerge after recovery from acute EAE; the rate at which this occurs may be connected to the peak severity of clinical disease. A return to pre-disease physiologic patterns after recovery may be normal in the SJL mouse, as EAE follows a remitting-relapsing disease course in this strain. Because this was a pilot study, we observed mice for only $17 \mathrm{~d}$. It is not known whether normal HR and BP patterns would be disrupted to the same extent with each relapse, or whether these patterns would be fully restored at each remission. Nor is it known whether or to what extent these physiological parameters might vary during chronic EAE. Studies with longer durations and larger group sizes are needed to investigate these questions, and our results suggest that such studies are feasible. Our results may be relevant to chronic fatigue, a common debilitating condition associated with MS, particularly because autonomic dysfunction, disturbances of the circadian rhythm and deregulation of hormonal signals associated with the sleep-wake cycle (all of which can affect HR and BP) are believed to contribute to chronic fatigue ${ }^{34,35}$.

Although the sympathetic and parasympathetic contributions to HRV have been known for some time ${ }^{1,2}$, investigations of autonomic influence on HRV in mouse models were not carried out until relatively recently because of limitations of monitoring on such a small scale $29,32,36$. Researchers have since been able to examine the effects of cardiac-associated mutations on HRV in various mouse models, including mice lacking the Mas proto-oncogene ${ }^{37}$, as well as transgenic mice with overexpression of a cardiac-specific GTPase ${ }^{32}$. To our knowledge, our present study is the first to investigate fluctuations in HRV related to the onset of autoimmune disease in a mouse model. Our analysis of HRV by frequency domain detected changes at both

TABLE 2 | Time domain analysis of HRV in mouse 4 during the progression of EAE

\begin{tabular}{|c|c|c|c|c|c|c|}
\hline Day & EAE score & $\mathrm{RR}(\mathrm{ms}) \pm \mathrm{s} . \mathrm{d}$ & SDRR & $P_{\text {SDRR }}$ & $\mathrm{CV}(\%) \pm \mathrm{s} . \mathrm{d}$ & $P_{\mathrm{CV}}$ \\
\hline 0 & 0 & $102 \pm 9$ & $5 \pm 1$ & - & $5 \pm 1$ & - \\
\hline 4 & 0 & $112 \pm 7$ & $5 \pm 3$ & 0.78 & $4 \pm 3$ & 0.4 \\
\hline 8 & 0 & $102 \pm 4$ & $4 \pm 1$ & 0.05 & $4 \pm 1$ & 0.06 \\
\hline 9 & 1 & $135 \pm 9$ & $10 \pm 4$ & 0.002 & $7 \pm 3$ & 0.04 \\
\hline 10 & 4 & $94 \pm 3$ & $3 \pm 0.5$ & $<0.0001$ & $3 \pm 0.4$ & $<0.0001$ \\
\hline 11 & 5 & $84 \pm 3$ & $2 \pm 0.5$ & $<0.0001$ & $2 \pm 0.6$ & $<0.0001$ \\
\hline 12 & 5 & $93 \pm 2$ & $3 \pm 1$ & 0.001 & $4 \pm 1$ & 0.01 \\
\hline 13 & 4 & $82 \pm 2$ & $2 \pm 0.5$ & $<0.0001$ & $2 \pm 0.6$ & $<0.0001$ \\
\hline 15 & 4 & $87 \pm 2$ & $3 \pm 1$ & 0.0005 & $4 \pm 1$ & 0.01 \\
\hline 17 & 4 & $94 \pm 5$ & $3 \pm 1$ & 0.001 & $3 \pm 1$ & 0.0002 \\
\hline
\end{tabular}


high and low spectral frequencies in mice with induced EAE; these findings may indicate the disruption of both sympathetic and parasympathetic influences ${ }^{29,32}$. Analysis by time domain also indicates that a disruption of autonomic control may occur in EAE mice. Further investigation is needed to determine whether HRV in EAE is a relevant parameter for studying autonomic dysfunction in MS, particularly chronic disease. Such studies may also provide insight into the involvement of cardiovascular disease and fatigue in MS.

Paralysis might cause a stress response that influences a loss of autonomic regulation; it is not known to what extent such an effect would occur. Stress hormones may initially contribute to higher HR and BP during paralysis in $\mathrm{EAE}^{38,39}$. This scenario, however, is not supported by our observation of diurnal pattern disruption before detecting any signs of paralysis ${ }^{40}$.

Although this topic has not been studied in depth, several reports indicate that circadian rhythm abnormalities may be associated with MS pathogenesis. A study of MS patients with fatigue, as mentioned earlier, found a significant correlation between fatigue and abnormal sleep patterns, indicating circadian rhythm disturbance ${ }^{34}$. Additional reports have found disturbances associated with the circadian control of core body temperature ${ }^{41,42}$. The importance of the circadian clock in maintaining a daily rhythm for metabolic and physiologic functions, including control of the autonomic nervous system, suggests that disruption of clock function could contribute to numerous chronic diseases ${ }^{43,44}$. Physiological monitoring of HR and BP in animal models may provide an approach for understanding the roles of such disruptions.

Finally, if we find that disturbances in HR and BP can be correlated with disease progression in EAE, it may be useful to monitor HRV, HR and BP as indicators of disease status or of therapeutic efficacy in MS. This could be informative, as our data indicate that a disruption of autonomic controls may occur before any overt paralytic signs of disease.

\section{ACKNOWLEDGMENTS}

This work was supported in part by the Office of Research and Development, Medical Research Service, Department of Veterans Affairs, and by the National Center for Complementary and Alternative Medicine (grant number AT002656).

\section{COMPETING INTERESTS STATEMENT}

The authors declare no competing financial interests.

\section{Received 1 February; accepted 11 April 2008}

Published online at http://www.labanimal.com/

1. Akselrod, S. et al. Power spectrum analysis of heart rate fluctuation: a quantitative probe of beat-to-beat cardiovascular control. Science 213, 220-222 (1981).

2. Pomeranz, B. et al. Assessment of autonomic function in humans by heart rate spectral analysis. Am. J. Physiol. 248, H151-H153 (1985).
3. Bigger, J.T. Jr. et al. Frequency domain measures of heart period variability and mortality after myocardial infarction. Circulation 85, 164-171 (1992).

4. Nolan, J. et al. Prospective study of heart rate variability and mortality in chronic heart failure: results of the United Kingdom heart failure evaluation and assessment of risk trial (UK-heart). Circulation 98, 1510-1516 (1998).

5. Heragu, N.P. \& Scott, W.A. Heart rate variability in healthy children and in those with congenital heart disease both before and after operation. Am. J. Cardiol. 83, 1654-1657 (1999).

6. Carnethon, M.R. et al. The association among autonomic nervous system function, incident diabetes, and intervention arm in the Diabetes Prevention Program. Diabetes Care 29, 914-919 (2006).

7. Routledge, H.C., Manney, S., Harrison, R.M., Ayres, J.G. \& Townend, J.N. Effect of inhaled sulphur dioxide and carbon particles on heart rate variability and markers of inflammation and coagulation in human subjects. Heart 92, 220-227 (2006).

8. Nance, D.M. \& Sanders, V.M. Autonomic innervation and regulation of the immune system (1987-2007). Brain Behav. Immun. 21, 736-745 (2007).

9. Sternberg, E.M. Neural regulation of innate immunity: a coordinated nonspecific host response to pathogens. Nat. Rev. Immunol. 6, 318-328 (2006).

10. Wrona, D. Neural-immune interactions: an integrative view of the bidirectional relationship between the brain and immune systems. J. Neuroimmunol. 172, 38-58 (2006).

11. Senaratne, M.P., Carroll, D., Warren, K.G. \& Kappagoda, T. Evidence for cardiovascular autonomic nerve dysfunction in multiple sclerosis. J. Neurol. Neurosurg. Psychiatry 47, 947-952 (1984).

12. Pentland, B. \& Ewing, D.J. Cardiovascular reflexes in multiple sclerosis. Eur. Neurol. 26, 46-50 (1987).

13. Flachenecker, P., Wolf, A., Krauser, M., Hartung, H.P. \& Reiners, K. Cardiovascular autonomic dysfunction in multiple sclerosis: correlation with orthostatic intolerance. J. Neurol. 246, 578-586 (1999).

14. Gunal, D.I., Afsar, N., Tanridag, T. \& Aktan, S. Autonomic dysfunction in multiple sclerosis: correlation with diseaserelated parameters. Eur. Neurol. 48, 1-5 (2002).

15. Flachenecker, P., Reiners, K., Krauser, M., Wolf, A. \& Toyka, K.V. Autonomic dysfunction in multiple sclerosis is related to disease activity and progression of disability. Mult. Scler. 7, 327-334 (2001).

16. Flachenecker, P. et al. Fatigue in MS is related to sympathetic vasomotor dysfunction. Neurology 61, 851-853 (2003).

17. Mahovic, D. \& Lakusic, N. Progressive impairment of autonomic control of heart rate in patients with multiple sclerosis. Arch. Med. Res. 38, 322-325 (2007).

18. Vita, G. et al. Cardiovascular autonomic dysfunction in multiple sclerosis is likely related to brainstem lesions. J. Neurol. Sci. 120, 82-86 (1993).

19. Acevedo, A.R., Nava, C., Arriada, N., Violante, A. \& Corona, T. Cardiovascular dysfunction in multiple sclerosis. Acta Neurol. Scand. 101, 85-88 (2000).

20. Saari, A. et al. Cardiovascular autonomic dysfunction correlates with brain MRI lesion load in MS. Clin. Neurophysiol. 115, 1473-1478 (2004).

21. Diamond, B.J., Kim, H., DeLuca, J. \& Cordero, D.L. Cardiovascular regulation in multiple sclerosis. Mult. Scler. $\mathbf{1}$ 156-162 (1995).

22. Monge-Argiles, J.A., Palacios-Ortega, F., Vila-Sobrino, J.A. \& Matias-Guiu, J. Heart rate variability in multiple sclerosis during a stable phase. Acta Neurol. Scand. 97, 86-92 (1998).

23. Sanya, E.0. et al. Abnormal heart rate and blood pressure responses to baroreflex stimulation in multiple sclerosis patients. Clin. Auton. Res. 15, 213-218 (2005).

24. Whitham, R.H. et al. Location of a new encephalitogenic epitope (residues 43 to 64 ) in proteolipid protein that induces relapsing experimental autoimmune encephalomyelitis in $\mathrm{PL} / \mathrm{J}$ 
and (SJL x PL)F1 mice. J. Immunol. 147, 3803-3808 (1991).

25. Kuchroo, V.K. et al. Induction of experimental allergic encephalomyelitis by myelin proteolipid-protein-specific T cell clones and synthetic peptides. Pathobiology 59, 305-312 (1991).

26. Kramer, K. \& Kinter, L.B. Evaluation and applications of radiotelemetry in small laboratory animals. Physiol. Genomics 13, 197-205 (2003).

27. Kramer, K. et al. Telemetric monitoring of blood pressure in freely moving mice: a preliminary study. Lab. Anim. 34, 272-280 (2000).

28. Desai, K.H. et al. Cardiovascular indexes in the mouse at rest and with exercise: new tools to study models of cardiac disease. Am. J. Physiol. 272, H1053-H1061 (2006).

29. Gehrmann, J. et al. Phenotypic screening for heart rate variability in the mouse. Am. J. Physiol. Heart Circ. Physiol. 279, H733-H740 (2000).

30. Li, P., Sur, S.H., Mistlberger, R.E. \& Morris, M. Circadian blood pressure and heart rate rhythms in mice. Am. J. Physiol. 276, R500-R504 (1999).

31. Chu, V. et al. Method for non-invasively recording electrocardiograms in conscious mice. BMC Physiol. 1 (2001).

32. Uechi, M. et al. Depressed heart rate variability and arterial baroreflex in conscious transgenic mice with overexpression of cardiac Gsalpha. Circ. Res. 82, 416-423 (1998).

33. Malik, M. \& Camm, A.J. Components of heart rate variabilitywhat they really mean and what we really measure. Am. J. Cardiol. 72, 821-822 (1993).

34. Attarian, H.P., Brown, K.M., Duntley, S.P., Carter, J.D. \& Cross, A.H. The relationship of sleep disturbances and fatigue in multiple sclerosis. Arch. Neurol. 61, 525-528 (2004).

35. Gold, S.M. et al. The role of stress-response systems for the pathogenesis and progression of MS. Trends Immunol. 26,
644-652 (2005).

36. Farah, V.M.A., Joaquim, L.J., Bernatova, I. \& Morris, M. Acute and chronic stress influence blood pressure variability in mice. Physiol. Behav. 83, 135-142 (2004).

37. Walther, T. et al. Altered heart rate and blood pressure variability in mice lacking the Mas protooncogene. Braz. J. Med. Biol. Res. 33, 1-9 (2000).

38. Dowdell, K.C., Gienapp, I.E., Stuckman, S., Wardrop, R.M. \& Whitacre, C.C. Neuroendocrine modulation of chronic relapsing experimental autoimmune encephalomyelitis: a critical role for the hypothalamic-pituitary-adrenal axis. J. Neuroimmunol. 100, 243-251 (1999).

39. Stefferl, A. et al. Disease progression in chronic relapsing experimental allergic encephalomyelitis is associated with reduced inflammation-driven production of corticosterone. Endocrinology 142, 3616-3624 (2001).

40. Andreini, I. et al. Function of the hypothalamo-pituitaryadrenal axis and humoral immune mechanisms during experimental allergic encephalomyelitis in $\mathrm{SJ} / \mathrm{J}$ mice. Neuroimmunomodulation 10, 9-16 (2002).

41. Sandyk, R. Diurnal variations in vision and relations to circadian melatonin secretion in multiple sclerosis. Int. J. Neurosci. 83, 1-6 (1995).

42. Martinez-Rodriguez, J.E., Munteis, E. \& Roquer, J. Periodic hyperthermia and abnormal circadian temperature rhythm in a patient with multiple sclerosis. Mult. Scler. 12, 515-517 (2006).

43. Hastings, M.H., Reddy, A.B. \& Maywood, E.S. A clockwork web: circadian timing in brain and periphery, in health and disease. Nat. Rev. Neurosci. 4, 649-661 (2003).

44. Maywood, E.S., O'Neill, J., Wong, G.K., Reddy, A.B. \& Hastings, M.H. Circadian timing in health and disease. Prog. Brain Res. 153, 253-269 (2006). 